\title{
SOME REMARKS ON ALMOST FINITELY GENERATED NILPOTENT GROUPS
}

\author{
Peter Hilton and Robert Militello
}

Dedicated to the memory of Pere Menal

\begin{abstract}
We identify two generalizations of the notion of a finitely generated nilpotent. Thus a nilpotent group $G$ is $f g p$ if $G_{p}$ is $f g$ as $p$-local group for each $p$; and $G$ is $f g$-like if there exists a $f g$ nilpotent group $H$ such that $G_{p} \cong H_{p}$ for all $p$. Then we have proper set-inclusions

$$
\{f g\} \subset\{f g \text {-like }\} \subset\{f g p\} .
$$

We examine the extent to which $f g$-like nilpotent groups satisfy the axioms for a Serre class. We obtain a complete answer only in the case that $[G, G]$ is finite. (The collection of $f g p$ nilpotent groups is known to form a Serre class in the extended sense).
\end{abstract}

\section{Introduction}

Recently (see [CH1], [CH2]) a certain natural generalization of the concept of a finitely generated $(f g)$ nilpotent group has been studied. Thus, given a prime $p$, we say that the $p$-local group $M$ is $f g$ as $p$-local group if there exists a $f g$ group $N$ such that $N_{p}=M$. We then say that the nilpotent group $G$ is $f g$ at every prime, or $f g p$, if $G_{p}$ is $f g$ as $p$-local group for every prime $p$. Plainly $f g$ groups are $f g p$, but the converse fails. It was shown in [H2] that $f g p$ groups form a Serre class in the extended sense of [HR].

Among the $f_{g p}$ (nilpotent) groups we may pick out those groups $G$ such that there exists a $f g$ group $H$ with $G_{p} \cong H_{p}$, all $p$. We then call $G f g$-like or, more specifically, $H$-like. It is plain that we have strict inclusions

$\{f g$ groups $\} \subset\{f g$-like groups $\} \subset\{f g p$ groups $\}$. 
For if $B$ is the subgroup of $Q$ generated by the rationals $\left(\frac{1}{p}\right.$, all $\left.p\right)$, then $B$ is not $f g$ but is plainly $\mathbb{Z}$-like; and if $A=\bigoplus_{p} \mathbb{Z} / p$ then $A$ is plainly $f g p$, but it cannot be $f g$-like since the torsion subgroup of any $f g$-like group must be finite.

Moreover, this example shows that the $f g$-like groups do not form a Serre class, since we have a short exact sequence.

$$
\mathbb{Z} \mapsto B \rightarrow A .
$$

However, for abelian groups, the only axiom which fails is that which asserts that a quoticnt group of a member of the class is again a member of the class. This we show in the next section, where we regard the Serre axioms relating to a short exact sequence as the principal axioms and the remaining axioms as subsidiary. However, the fact that a subgroup of an abelian $f g$-like group is $f g$-like, and the fact that an abelian extension of an $f g$-like group by an $f g$-like group is $f g$-like, both follow from an easy characterization of abelian $f g$-like groups as those abelian $f g p$ groups whose torsion subgroups are finite. For nilpotent groups we do not know if this characterization holds; certainly $f g$-like nilpotent groups have finite torsion subgroups, but we do not know whether $f g p$ nilpotent groups with finite torsion subgroups are necessarily $f g$-like. If the characterization held then the corresponding Serre axioms for $f g$-like nilpotent groups could be proved just as easily as in the abelian case.

In Section 3 we go as far as we can in the nilpotent case. Of course, the homology axiom holds; for the homology groups of a $f g$ nilpotent group are $f g$, and, if $G$ is $K$-like, with $K f g$, then $H_{k}(G)$ is $H_{k}(K)$-like. As to the principal axioms, one of couse is false and, as to the others, we are only able to prove them in the case of those groups $G$ such that $[G, G]$ is finite. This we do by means of our main theorem, Theorem 3.1, which asserts that if $Q=G / N$ with $N$ finite, then $G$ is $f g$-like if and only if $Q$ is $f g$-like. From this it readily follows (Theorem 3.5 ) that if $G$ is $f g p$ with $T G$ finite and if $[G, G]$ is finite, then $G$ is $f g$-like.

In an Appendix we show that Schur's Theorem, namely, that if $G$ is a group with $G / Z G$ finite, where $Z G$ is the center of $G$, then $[G, G]$ is finite, admits a converse if $G$ is nilpotent $f g p$. Moreover, precisely the same primes enter into the orders of $G / Z G$ and $[G, G]$.

\section{The Abelian case}

We have already observed that a quotient of an $f g$-like abelian group need not be $f g$-like. We will show in this section that this is the only axiom for a Serre class which fails. As to the subsidiary axioms, this follows from the following composite theorem. 
Theorem 2.1. Let $A$ be $M$-like and $B$ be $N$-like (where all groups are abelian). Then

(i) $A \otimes B$ is $M \otimes N$-like.

(ii) $\operatorname{Tor}(A, B) \cong \operatorname{Tor}(M, N)$.

(iii) $H_{k}(A)$ is $H_{k}(M)$-like.

Proof:

(i) We have, for any prime $p,(A \otimes B)_{p}=A_{p} \otimes B_{p} \cong M_{p} \otimes N_{p}=$ $(M \otimes N)_{p}$.

(ii) Similarly, for any prime $p, \operatorname{Tor}(A, B)_{p} \cong \operatorname{Tor}(M, N)_{p}$; but $\operatorname{Tor}(A, B)$ is a torsion group, so $\operatorname{Tor}(A, B) \cong \operatorname{Tor}(M, N)$.

(iii) We have, for any prime $p$, and $k \geq 1, H_{k}(A)_{p}=H_{k}\left(A_{p}\right) \cong$ $H_{k}\left(M_{p}\right)=H_{k}(M)_{p}$.

If $k=0$, the assertion is trivial.

As to the principal axioms, we have only to prove (a) that a subgroup of an $f g$-like abelian group is $f g$-like; and (b) that an abelian extension of an $f g$-like group by an $f g$-like group is $f g$-like. We base these results on the following obvious characterization of abelian $f g$-like groups. (Compare Theorem 1.3 of [CH2]).

Proposition 2.2. Let $A$ be an abelian group. Then $A$ is fg-like if and only if $A$ is fgp and $T A$ is finite.

Proof: If $A$ is $M$-like with $M f g$, then $T A \cong T M$ which is evidently finite. Thus if $A$ is $f g$-like, then $A$ is $f g p$ and $T A$ is finite. Conversely, suppose that $T A$ is finite and $A$ is $f g p$. Then $F A$ is $\mathbb{Z}^{k}$-like, for some $k$, so that (sec Theorem 1.1 of [CH2]) $\operatorname{Ext}(F A, T A)=0$ and $A=T A \oplus F A$. It follows that $A$ is $M$-like, where $M=T A \oplus \mathbb{Z}^{k}$.

\section{Corollary 2.3.}

(a) A subgroup of an $f g$-like abelian group is $f g$-like.

(b) An abelian extension of an fg-like group by an fg-like group is fg-like.

Proof: Since $f g p$ abelian groups form a Serre class [H2], it remains only, in the light of Proposition 2.2, to show that the property of having a finite torsion subgroup is preserved by subgroups and extensions. This is absolutely clear for subgroups. For extensions, we observe that, if

$$
G^{\prime} \mapsto G \stackrel{\pi}{\rightarrow} G^{\prime \prime}
$$

is exact, so is

$$
T G^{\prime} \frown T G \rightarrow \pi(T G)
$$


and $\pi(T G) \subseteq T G^{\prime \prime}$. Thus, if $T G^{\prime}$ and $T G^{\prime \prime}$ are finite, so is $T G$.

Remark. We notice that the proof of Corollary 2.3 works in the nilpotent case, provided the characterization of Proposition 2.2 remains valid for nilpotent groups. Certainly $f g$-like nilpotent groups are $f g p$ with finite torsion subgroups, but we have not been able to establish the converse. Thus we have also not succeeded in generalizing Corollary 2.3 to nilpotent groups.

\section{The Nilpotent case}

Our basic result in the nilpotent case is the following.

Theorem 3.1. Let $N \mapsto G \rightarrow Q$ be a short exact sequence of nilpotent groups with $N$ finite. Then $G$ is fg-like if and only if $Q$ is fg-like.

Proof: Suppose $G$ is $K$-like, with $K f g$. We may then assume that $T G=T K$ and that, $\forall p, G_{p}=K_{p}$. Now $N \subseteq T G=T K \subseteq K$ and $N_{p}$ is normal in $K_{p}\left(=G_{p}\right)$ for all $p$. It thus follows from [HM] that $N$ is normal in $K$. Set $L=K / N$, so that $L$ is $f g$. For all $p$, we have

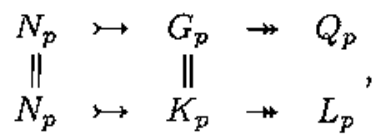

yiclding isomorphisms $Q_{p} \cong L_{p}$, so that $Q$ is $L$-like.

Conversely, suppose that $Q$ is $L$-like, with $L f g$; and suppose further that $N$ is commutative. Then the sequence $N \rightarrow G \rightarrow Q$ determines a nilpotent action of $Q$ on $N$, and then the extension represents an element $\eta=[G] \in H^{2}(Q ; N)$, where $N$ is regarded as a (nilpotent) $Q$-module. Now there are associated nilpotent actions of $Q_{p}$ on $N_{p}$, for all $p$, such that

$$
H^{2}(Q ; N)=H^{2}\left(Q ; \prod_{p} N_{p}\right)=\prod_{p} H^{2}\left(Q ; N_{p}\right) \cong \prod_{p} H^{2}\left(Q_{p} ; N_{p}\right)
$$

and, under this isomorphism, $\eta$ corresponds to $\left\{\eta_{p}\right\}$, where $\eta_{p} \in H^{2}$ $\left(Q_{p} ; N_{p}\right)$ is represented by

$$
N_{p} \mapsto G_{p} \rightarrow Q_{p}
$$

We may, as before, assume that $L_{p}=Q_{p}$ and then we let $L$ act on $N_{p}$ via $e_{p}: L \rightarrow L_{p}$. This action is, of course, nilpotent, and so is the 
induced action of $L$ on $N=\prod_{p} N_{p}$. Thus we obtain, paralleling (3.1), an isomorphism

$$
H^{2}(L ; N) \cong \prod_{p} H^{2}\left(L_{p} ; N_{p}\right)\left(=\prod_{p} H^{2}\left(Q_{p} ; N_{p}\right)\right)
$$

The isomorphisms (3.1), (3.2) compose to an isomorphism $H^{2}(Q ; N) \cong$ $H^{2}(L ; N)$, under which $\eta$ corresponds to, say, $\zeta$, where $\zeta$ is represented by the sequence $N \rightarrow K \rightarrow L$. Then $K$ is nilpotent and certainly $f g$. Moreover, for each $p$, we have an isomorphism

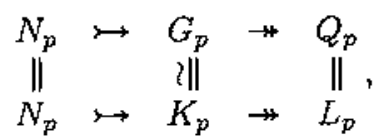

showing that $G$ is $K$-like.

We now complete the converse argument by induction on nil $N$. For the sequence $N \mapsto G \rightarrow Q$ gives rise to a sequence

$$
N /[N, N] \mapsto G /[N, N] \rightarrow Q
$$

so that, by what we have just proved, $G /[N, N]$ is $f g$-like. Finally we consider the sequence

$$
[N, N] \mapsto G \rightarrow G /[N, N] .
$$

Since $[N, N]$ is finite with nil $[N, N]<\operatorname{nil} N$, our inductive hypothesis allows us to infer that $G$ is $f g$-like.

Remark. The abelian version of Theorem 3.1 is trivial. For then Corollary 2.3(b) assures us that $G$ is $f g$-like if $Q$ is $f g$-like; and, in the other direction, the finiteness of $N$ tells us that $T G$ maps onto $T Q$, so $Q$ is $f g$-like by Proposition 2.2 .

Corollary 3.2. Let $G$ be a nilpotent group with finite commutatorsubgroup. Then $G$ is $f g$-like if and only if $G_{a b}$ is $f g$-like.

Proof: Of course we need no assumption on $[G, G]$ to infer that $G_{a b}$ is $f g$-like if $G$ is $f g$-like; for if $G$ is $K$-like, then $G_{a b}$ is $K_{a b}$-like. However, in the other direction, we must apply Theorem 3.1 .

Theorem 3.3. Let $G$ be fg-like and let $H \subseteq G$ be a subgroup such that $H \cap[G, G]$ is finite. Then $H$ is $f g$-like. In particular, all subgroups of $G$ are $f g$-like if $[G, G]$ is finite. 
Proof: Since $G$ is $f g$-like so is $G_{a b}$. Then $H / H \cap[G, G] \subseteq G_{a b}$, so that, by Corollary $2.3(\mathrm{a}), H / H \cap[G, G]$ is $f g$-like. We now use Theorem 3.1 to infer that $H$ is itself $f g$-like.

Theorem 3.4. Let $G^{\prime} \rightarrow G \rightarrow G^{\prime \prime}$ be a short exact sequence of nilpotent groups in which $G^{\prime}, G^{\prime \prime}$ are fg-like. Then, if $[G, G]$ is finite, $G$ is fg-like.

Proof: We have the short sequence of abelian groups

$$
G^{\prime} / G^{\prime} \cap[G, G] \nrightarrow G_{a b} \rightarrow G_{a b}^{\prime \prime}
$$

Moreover, $G_{a b}^{\prime \prime}$ and $G^{\prime} / G^{\prime} \cap[G, G]$ are $f g$-like, the latter by Theorem 3.1 . Thus, by Corollary $2.3(\mathrm{~b}), G_{a b}$ is $f g$-like. Hence, by Corollary $3.2, G$ is $f g$-like.

Remark. Note that the class of nilpotent groups with finite commutatorsubgroup is not closed under (nilpotent) extensions. Thus if $\Gamma$ is the free nilpotent group of class 2 on 2 generators, then $\Gamma$ has a central subgroup $[\Gamma, \Gamma]$ which is free cyclic with quotient free abelian of rank 2.

Theorems 3.3 and 3.4 suggest that, in the study of $f g$-like groups, nilpotent groups $G$ such that $[G, G]$ is finite behave very much like abelian groups (which may be regarded as special cases). In the light of Proposition 2.2 , this view is reinforced by the following theorem.

Theorem 3.5. Suppose $G$ is nilpotent with $[G, G]$ finite. Then $G$ is fg-like if and only if $G$ is fgp with $T G$ finite.

Proof: As remarked in the Introduction, if $G$ is $f g$-like then, even without the hypothesis that $[G, G]$ is finite, $G$ is $f g p$ with $T G$ finite. Suppose conversely that $G$ is $f g p$ with $T G$ finite and that $[G, G]$ is finite. Consider the short exact sequence.

$$
T G \mapsto G \rightarrow F G
$$

Since $[G, G]$ is finite, $[G, G] \subseteq T G$, so that $F G$ is abelian. Thus $F G$ is torsionfree, abelian and $f g p$, which implies that $F G$ is $\mathbb{Z}^{k}$-like for some integer $k \geq 0$. The conclusion now follows from Theorem 3.1 (or Theorem 3.4).

Of course, as hinted in the Introduction, it is also not difficult to deduce Theorems 3.3 and 3.4 from Theorem 3.5 . 


\section{Appendix: Groups with finite commutatorsubgroup}

A famous theorem due to I. Schur asserts that if $G / Z G$ is finite, then $[G, G]$ is finite. A study of its proof (we give a homological proof below) shows that more is true. Given any finite group $N$, let $\tau(N)$ be the set of primes dividing the order of $N$. Then we may conclude that $\tau[G, G] \subseteq \tau(G / Z G)$. In the context of nilpotent groups, we have a stronger result.

Theorem 4.1. Let $G$ be an fgp nilpotent group. Then $G / Z G$ is finite if and only if $[G, G]$ is finite and then $\tau(G / Z G)=\tau[G, G]$.

Proof: We first prove Schur's Theorcm. Thus if $G / Z G$ is finite then $G^{\prime} / G^{\prime} \cap Z G$ is finite, where $G^{\prime}=[G, G]$, and $\tau\left(G^{\prime} / G^{\prime} \cap Z G\right) \subseteq \tau(G / Z G)$. Thus it remains to prove that $G^{\prime} \cap Z G$ is finite, with $\tau\left(G^{\prime} \cap Z G\right) \subseteq$ $\tau(G / Z G)$. Now $\mathrm{H}_{2}(G / Z G)$ is finite with $\tau\left(H_{2}(G / Z G)\right) \subseteq \tau(G / Z G)$. Moreover there is an exact sequence

$$
H_{2}(G / Z G) \longrightarrow Z G \longrightarrow G_{a b}
$$

which shows that $G^{\prime} \cap Z G$ is a homomorphic image of $H_{2}(G / Z G)$. We conclude that $G^{\prime} \cap Z G$ is finite with $\tau\left(G^{\prime} \cap Z G\right) \subseteq \tau(G / Z G)$, as required. Indeed, we have shown that $\tau[G, G] \subseteq \tau(G / Z G)$, as claimed.

Conversely, suppose that $G$ is an $f g p$ nilpotent group with $G^{\prime}$ finite. Set $\tau=\tau\left(G^{\prime}\right)$. Then, for all $g, h \in G,[g, h]^{n}=1$ for some $\tau$-number $n$. If $b=[g, h]$, we have $g b=h^{-1} g h$ so that, by Corollary 6.2 of $[\mathbf{H 1}]$, $g^{n^{c}}=\left(h^{-1} g h\right)^{n^{c}}=h^{-1} g^{n^{c}} h$, where nil $G \leq c$. Thus $g^{n^{c}} \in Z G$, so that $G / Z G$ is a torsion group with bounded exponent dividing $n^{c}$. But $G$ is $f g p$, so $G / Z G$ is $f g p$. It follows that each $(G / Z G)_{p}$ is finite and $(G / Z G)_{p}$ is trivial unless $p \mid n^{c}$. We conclude that $G / Z G$ is a finite group with $\tau(G / Z G) \subseteq \tau=\tau[G, G]$. This completes the proof of the theorem.

\section{References}

[CH1] Casacuberta, Carles and Hilton, Peter, On nilpotent groups which are finitely generated at every prime, Expositiones Mathematicae (1991) (to appear).

[CH2] Casacuberta, Carles and Hitton, Peter, On special nilpotent groups, Bull. Soc. Mat. Belg. (Série B) Tome XLI, 3 (1989), 261-273. 
[HI] Hilton, Peter, Localization and cohomology of nilpotent groups, Math. Zeits. 132 (1973), 263-286.

[H2] HiltoN, PeTER, On a certain Serre class of nilpotent groups, Journ. of Algebra (1992) (to appear).

[HM] Hilton, PETER and Mislin, Guido, Bicartesian squares of nilpotent groups, Comm. Math. Helv. 50 (1975), 477-491.

[HR] Hilton, PEter and RoItberg, Joseph, Generalized $C$-theory and torsion phenomena in nilpotent spaces, Houston Journ. Moth. 2 (1976), 525-559.

Peter Hilton:

Department of Mathematical Sciences

State University of New York at Binghamton

New York

U.S.A.
Robert Militello:

Department of Mathematics

Rhodes College, Memphis

Tennessee

U.S.A.

Rebut el 18 de Desembre de 1991 